\title{
Detection of Breast Abnormalities of Thermograms based on a New Segmentation Method
}

\author{
Mona A. S. Ali ${ }^{4, *}$, Gehad Ismail Sayed ${ }^{5, *}$, Tarek Gaber ${ }^{1,2, *}$, \\ Aboul Ella Hassanien ${ }^{5, *}$, Vaclav Snasel ${ }^{3}$, Lincoln F. Silva ${ }^{6}$ \\ ${ }^{1}$ Faculty of Computers and Informatics, Suez Canal University, Egypt \\ ${ }^{2}$ IT4Innovations, VSB-TU of Ostrava, Czech Republic \\ ${ }^{4}$ Faculty of Computers and Information,Minia University, Egypt \\ ${ }^{5}$ Faculty of Computers and Information, Cairo University, Egypt \\ ${ }^{3}$ FEECS, Department of Computer Science and IT4Innovations, VSB-TU of Ostrava, Czech Republic \\ ${ }^{6}$ Department of Computer Science, Fluminense Federal University, Brazil \\ * Scientific Research Group in Egypt, (SRGE), http://www.egyptscience.net
}

\begin{abstract}
Breast cancer is one from various diseases that has got great attention in the last decades. This due to the number of women who died because of this disease. Segmentation is always an important step in developing a CAD system. This paper proposed an automatic segmentation method for the Region of Interest (ROI) from breast thermograms. This method is based on the data acquisition protocol parameter (the distance from the patient to the camera) and the image statistics of DMR-IR database. To evaluated the results of this method, an approach for the detection of breast abnormalities of thermograms was also proposed. Statistical and texture features from the segmented ROI were extracted and the SVM with its kernel function was used to detect the normal and abnormal breasts based on these features. The experimental results, using the benchmark database, DMR-IR, shown that the classification accuracy reached $(100 \%)$. Also, using the measurements of the recall and the precision, the classification results reached $100 \%$. This means that the proposed segmentation method is a promising technique for extracting the ROI of breast thermograms.
\end{abstract}

\section{INTRODUCTION}

B REAST cancer is the most common one among women, and it most likely cause of women death in the world wide. In the United States, one death of 4 is due to cancer [1]. There are many breast imaging techniques used for identifying early stage of breast cancer. One of them is mammography. It is most commonly used for screening of breast cancer. However the false negative rate can reach up to $30 \%$ in addition it expose patient to ionizing radiation effect [2]. Thermography uses a special heat-sensing camera to measure and map the heat on the surface of the breast [3], [4].

As long as cancerous tumors have an increased blood supply and cell growth they tend to be warmer than the surrounding normal tissue and it is the idea behind using Thermal images instead mammogram. Thermography play an important rule as a visualization technique that can be used in many different fields of physics and science. Unlike mammography, there is no compression of the breast so women may find it more comfortable, thermal imaging does not expose the woman to any radiation, as occurs with mammography (a type of X-ray), fast, low cost and sensitive method [5].
The extraction of the Region Of Interest, ROI segmentation, from the breast image is very important for detecting the cancer from the breast images. ROI segmentation aims to separate the regions of the breast from the other parts of the body. It can range from a completely manual to a fully automatic process [6]. Once the breast region is separated from the rest of thermal images, then some features are extracted. Then some kindS of artificial classifications algorithms are applied to classify the organs in analysis as normal or abnormal [7].

For the automatic segmentation approaches [7], [8], the level set technique [9] used to extract the blood vessels in a thermal image. The level set function is evolved using the gradient magnitude and direction of an edge map provided by few initial points selected in region of interest. In [7], automatic segmentation approach is proposed using active contour and level set method without re-initialization. It is used to extract the breast regions from breast thermograms. Before applying the level set, a statistical based noise removal and contrast-limited-adaptive histogram equalization are used to improve signal to noise ratio and the contrast of thermal images. Verification and validation of the segmented results are carried out using 60 images against the ground truths. The segmented areas are observed to be in good correlation with the ground truth areas as the correlation coefficient was $98 \%$.

For breast abnormalities, several attempts [10], [11], [12], [13], [14] have been made to demonstrate the ability of different features in detecting breast cancer abnormalities. Texture features have been used to detect abnormal thermograms using support vector machine (SVM) [11] and artificial neural networks [12]. Statistical features ranging from first order statistical to higher order statistical features have been extracted for automatic classification of abnormal breast conditions using different classifiers like SVM [13], linear discriminant classifier, minimum distance classifier and Parzen window [14].

This paper proposes an automatic breast segmentation approach based on the distance between body and camera is 1 meter (dynamic protocol). In order to evaluate the accuracy of the proposed segmentation approach, different selective 
features were extracted from the segmented regions and then the SVM classifier was used to detect the breast abnormalities

This paper is organized as follows. Section II reviews the existed segmentation and the extracted features methods. Section III gives overview of the thermal imaging protocols of breast cancer while Section IV presents the proposed approach to segment region of interest. In Section V the proposed segmentation is used to detect the abnormalities of thermogarms images and IN Section VII the experimental results are discussed. Finally, we conclude the paper and gives some future directions in Section VIII.

\section{LITERATURE REVIEW}

To make our proposed approach, in this paper comparable with its related work, we have limited this related work to the efforts done using the DMR-IR database [15]. These efforts can be classified into two classes: automatic segmentation of breast regions [7]] [8] and classification based on the asymmetry analysis to normal and abnormal cases [16], [17], [18].

For the automatic segmentation [7], [8], the level set technique [9] has been used to extract the blood vessels in a thermal image. The Level set function was evolved using the gradient magnitude and direction of an edge map provided by few initial points selected in region of interest. In [7], an automatic segmentation approach, using active contour and level set method without re-initialization, was proposed to extract the breast regions from breast thermograms. Before applying the level set, a statistical based noise removal technique and contrast limited adaptive histogram equalization were used to improve signal to noise ratio and contrast of thermal images. Verification and validation of the segmented results were carried out using 60 images against the ground truths. The segmented areas were observed to be in good correlation with the ground truth areas as the correlation coefficient was 98

Another automatic segmentation approach has been proposed in [8] to segment the frontal breast tissues from breast thermograms. This approach made use of the Modified Phase Based Distance Regularized Level Set (MPBDRLS) method. The method was further modified by adopting an improved diffusion rate model. The segmented region of interests was evaluated using 72 gray scale images of size $320 \times 240$ pixels and against the ground truth images. The overlap measures showed that the average similarity between four sets ground truths and segmented region of interests was $97 \%$.

The asymmetric-based classification is based on the asymmetric abnormalities which can be identified by comparing the features extracted from the breast regions (right and left). Several statistical and fractal features are found to be useful features in identification of pathological conditions of breast tissues [18]. Using DMR-IR database, in [16], an approach was proposed to classify the normal and abnormal (carcinoma, nodule and fibro adenoma) breast thermograms Gabor wavelet transform. First, the segmentation of the breast tissues was performed using ground truth masks and the raw images. Gabor features [19] were then extracted for the detection of the abnormalities. The results showed that from total of 20 images, used of the approach evaluation, there were 9 images with carcinomas, 6 with nodules, and 5 with fibro adenomas.

\section{INFRARED IMAGING PROTOCOLS}

Infra-red imaging protocols in general have the following parts: Recommendations to the patient, conditions of the examination room; preparation, cooling and positioning of the patient; capturing positions and parameters. For recommendation to the patient, patient asked to avoid: Alcohol, caffeine, physical exercises, and nicotine for at least two hours before the examination process. And for condition of examination room, Room temperature must be maintained between $20^{\circ} \mathrm{C}$ and $22{ }^{\circ} \mathrm{C}$, no airflow directed to the patient no windows, no opening.

For preparation of the patient, she is asked to remove earrings, necklaces or other accessory. The patient's body temperature is checked by clinical thermometer and her hair stuck with a cap. The patient is positioned in front of the camera with the hands on the head. And for capturing positioning and parameters, the standard distance between the camera and the patient is $1 \mathrm{~m}$. But it depend on the size of the patient, a distance of $0.8 \mathrm{~m}$ or $1.2 \mathrm{~m}$ is adopted for a better frame of the region of interest (breast and armpit) in the image. The distance, room temperature and the relative humidity of the air are recorded and inserted as parameters in the IR camera settings [20].

In all reproduced protocols, the initial procedures are equal to each volunteer. These procedures are i) to check the central temperature by a clinical thermometer; ii) to check whether the volunteer followed the recommendations of the protocol which are at least two hours before: iii) to ask the patient to remove beads, earrings and other accessories which can be viewed in the thermal images and hold the hair with a shower cap; and iv) to ask the patient to remove clothing from the waist up.

In all protocols below the volunteer stays with his hands on his head during the whole process.

1) First Static Protocol: The volunteer rests for 10 minutes to stabilize the skin temperature of the breasts and armpit. After that, a frontal image is captured.

2) Second Static Protocol: Similar procedures of the previous protocol, but in this, the volunteer rests per 15 minutes.

3) First Dynamic Protocol: An electric fan is turned on and directed to the volunteer's breasts and armpits per 2 minutes. After this, six images are captured, one each minute.

4) Second Dynamic Protocol: Alcohol is applied at the region of the volunteer's breasts and armpits, then an electric fan is turned on and guided to this region per 30 seconds. After this, six images are captured, one each minute. 


\section{The New Segmentation Method}

The proposed segmentation method depends on the predefined parameter (i.e. th distance between camera and patient) of the data acquisition protocol used to collect thermal breast image in [20]. In our case, the breast's region occupies nearly half of the image height while the other areas include women shoulders and stomach. Figure 1 confirm these facts.

The main idea of the proposed segmentation method is based on the following facts [20]:

1) The distance between body and camera is 1 meter (dynamic protocol).

2) As can be seen from Figure 1, the image includes only the upper part of the patient that contains a part of the stomach and arms with nick.

3) The breast engaged a specific position in human body which is nearly at the center of the image, see Figure 1 .

As a result of these facts, we followed the algorithm as see in Algorithm (1).

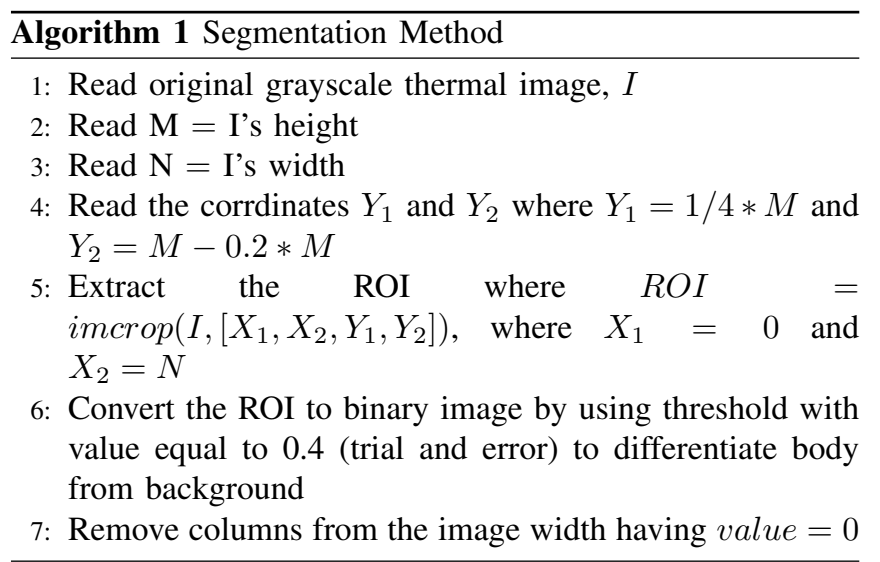

In case, as a women body is not always at center of image then after getting initial ROI extracted, threshold will be used to binaries the image. Then full column with zero value will be removed in order to focus on breast region. Figures 3 and 2 show the extracted ROI from original grayscale thermal image in case of normal and abnormal.

To explain how this new segmentation method works, we give the following example. Suppose, there is an image with 480 height and 640 width as seen in Figure 4. Based on Algorithm (1), the coordinate of the ROI are $X$ where $X$ ranges form 1 to 640 and $Y$ where $Y$ from 120 to 384 .

\section{BReAst ABNormalities Detection ApproACH}

To evaluate the new segmentation method, we proposed a breast abnormalities detection approach. As shown in Figure 5, this approach consists of four phases: ROI segmentation, ROI enhancement, feature extraction and classification.

a) ROI Segmentation: : At this phase, our method, introduced in Section (IV) will be applied to extract the ROI (i.e. the breast region) from original grayscale thermal image. b) ROI Enhancement: Image enhancement is important step in image processing. It used to bring out detail that is obscured, or simply to highlight certain features of interest in an image. Then histogram equalization is used to enhance contrast of the image and increase classification accuracy.

c) Features Extraction: Two types of features (first order statistical and texture) are extracted from the enhanced ROI of breast thermograms. The first order statistical features include mean, standard deviation, median, mode, skewness and kurtosis whereas the texture ones consists 15 features which were extracted from gray-level co-occurrence matrix (GLCM) [21] with the distance parameter $d=1$. These features are Energy, Contrast, Correlation, Sum of square variance, Homogeneity, Dissimilarity, Inverse difference moment (IDM), Inverse difference normalized (INN), Information measure of correlation1 (IMC1), Information measure of correlation2 (IMC2), Difference entropy, Difference variance, Sum entropy, Sum variance and Sum average. So, the total extracted features is 21 features.

Table I and Table II show the features extracted from the ROI of both normal an abnormal cases. To increase the performance of the proposed approach, before using these features in the classification phase, they were analyzed using student's $t$ hypothesis testing the mean of two independent samples. The features with $P>0.05$ were selected for the classification process. Based on this test, as seen Table I, the Median, Mode and Standard deviation are statistically insignificant where $P>0.05$ whereas the Mean, Skewness and Kurtosis are found to be statistically significant where $P<0.05$. Also, form Table II Homogeneity and Information Measure of Correlation1 (IMC1) are found insignificant features and the Energy, Contrast, Correlation, Sum of square variance, Dissimilarity, Inverse difference moment (IDM), Inverse difference normalized (INN), Information measure of correlation2 (IMC2), Difference entropy, Difference variance, Sum entropy, Sum variance and Sum average were found highly significant features where $P<0.05$. Therefore, only 16 out of 21 features were used in the classification phase.

TABLE I: First order statistical features analysis for normal group (NG) and abnormal (AbG) group

\begin{tabular}{|c|c|c|c|}
\hline Feature & Average of NG & Average of AbG & p \\
\hline Mean & 105.622 & 123.905 & 0.023 \\
\hline Median & 110.485 & 126.742 & 0.061 \\
\hline Mode & 59.885 & 68.571 & 0.581 \\
\hline Standard Deviation & 14.123 & 16.169 & 0.089 \\
\hline Skewness & 0.8105 & 1.8128 & 0 \\
\hline Kurtosis & 15.245 & 31.415 & 0 \\
\hline
\end{tabular}

d) Classification: The Support vector machine (SVM) were used to evaluate the feature extracted from the ROI. The SVM is a supervised learning method that transforms input data to high-dimensional feature space using various kernel functions (Linear, polynomial, RBF, and quadratic) such that the transformed data becomes more separable that that of the original input data [22][23][24]. The SVM was first trained with the selected 16 features (i.e. features with $p<0.05$ ) to 

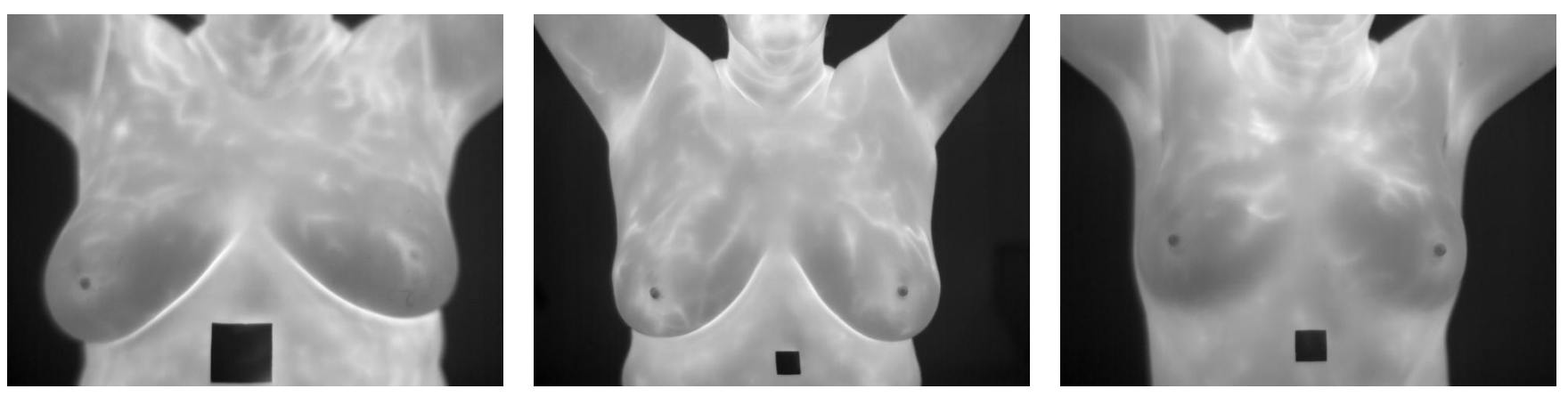

Fig. 1: Original Patient Image

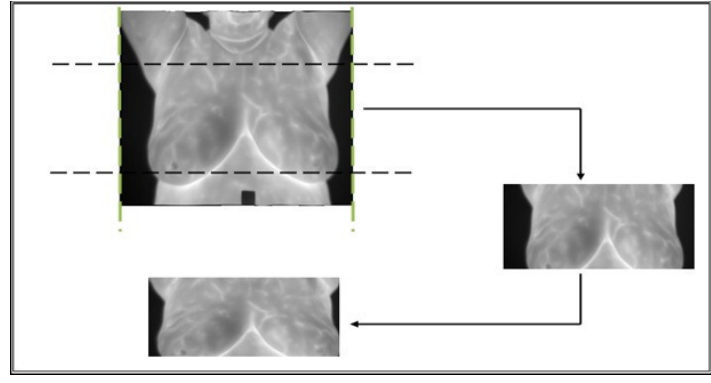

Fig. 2: Abnormal Segmentation Case

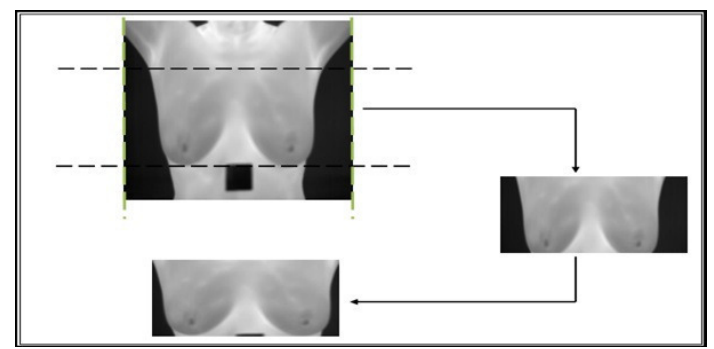

Fig. 3: Normal Segmentation Case

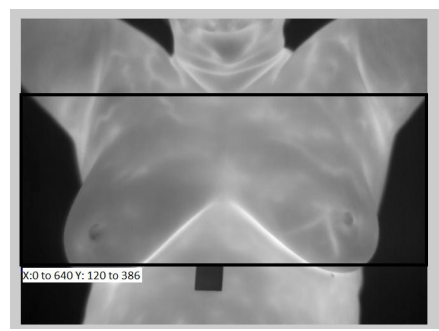

Fig. 4: Segmented ROI

build a classification model which was then used in the testing phase to classify breast image to normal or abnormal.

e) Support Vector Machine (SVM): SVM is one of the classifiers, which deals linearly or non-linearly to classify unknown objects based on increase the margin between classes. It deals successfully with high dimensional datasets ([19], [25]).

Given a training dataset or a feature matrix, $x_{i}$ and $y_{i}$, where $i=1,2,3, \ldots, N, N$ represents the number of training

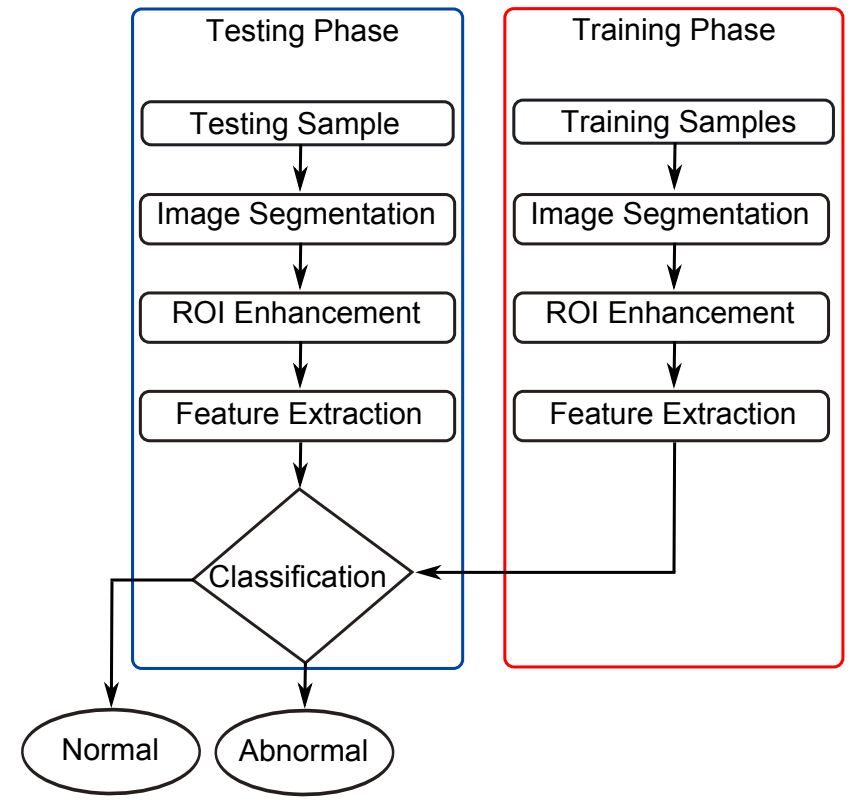

Fig. 5: Proposed Approach Model

samples, $x_{i}$ is a feature vector, and $y_{i}$ represent the class labels of the training sets. In case of binary classification $y_{i} \in\{-1,+1\}$ is the target label, $y=+1$, for samples belong to class $C_{1}$ and $y=-1$ denotes to samples belong to class $C_{2}$. The default SVM deals linearly with the classification problem, but using different kernels it may solve the problem of non-linear classification [19]. SVM algorithm tries to find an optimal hyperplane with the maximal margin to separate two different classes, which requires solving the optimization problem in Equation (1).

$$
\begin{array}{r}
\text { maximize } \sum_{i=1}^{n} \alpha_{i}-\frac{1}{2} \sum_{i, j=1}^{n} \alpha_{i} \alpha_{j} y_{i} y_{j} . K\left(x_{i}, x_{j}\right) \\
\text { subject to: } \sum_{i=1}^{n} \alpha_{i} y_{i}, 0 \leq \alpha_{i} \leq C
\end{array}
$$

where, $\alpha_{i}$ represents the weight assigned to the training sample $x_{i}$ (if $\alpha_{i}>0$, then $x_{i}$ is called a support vector); $C$ is a 
TABLE II: Second order statistical features analysis for normal group (NG) and abnormal (AbG) group

\begin{tabular}{|c|c|c|c|}
\hline Feature & Average of NG & Average of AbG & $\mathbf{p}$ \\
\hline Energy & 0.0025 & 0.0027 & 0.044 \\
\hline Contrast & 0.0369 & 0.047 & 0.013 \\
\hline Correlation & 0.7951 & 0.9288 & 0.028 \\
\hline Sum of Square variance & 18496 & 21703 & 0.024 \\
\hline Homogeneity & 0.2828 & 0.3108 & 0.236 \\
\hline Dissimilarity & 9.2618 & 11.448 & 0.004 \\
\hline IDM & 0.8264 & 0.9652 & 0.024 \\
\hline INN & 0.7967 & 0.9322 & 0.0255 \\
\hline IMC2 & 0.8169 & 0.9681 & 0.0244 \\
\hline IMC1 & -0.373 & -0.4241 & 0.0971 \\
\hline Difference Entropy & 1.969 & 2.3652 & 0.0078 \\
\hline Difference Variance & 369.02 & 470.77 & 0.0013 \\
\hline Sum Entropy & 4.15 & 4.8719 & 0.0215 \\
\hline Sum Variance & 7147.6 & 8377.8 & 0.0232 \\
\hline Sum Average & 214.7 & 251.8 & 0.0226 \\
\hline
\end{tabular}

regulation parameter; and $K$ is a kernel function, which is used to map or transform the features into higher dimensional space to discriminate between different samples

\section{EXPERIMENTAL RESULTS}

\section{A. Breast cancer Dataset}

A benchmark database [20] used to evaluate our proposed approach. This public database are constructed by collecting the IR images from UFF University's Hospital and publicly published under the approval of the ethical committee where every patient should sign consent. 63 IR single breast images $(640 \times 480$ pixels $)$ from this database were used in this paper (29 healthy and 34 malignant).

\section{B. Experimental Results}

This section presents two type of the results: segmentation results obtained by our new segmentation method and the classification results based on this new method.

a) Segmentation Results: Due to the fact that there are different size of the breast of each woman and this is the case of the DMR-IR database [20], four scenarios ( small size breast, medium size breast, large size breast, and the asymmetric breast size) were designed to evaluate our new segmentation method against these different cases. The segmentation results of all these scenarios using our new method are shown in Figure 6 which shows that automatic segmentation of the ROI of thermograms under the various image cases.

1) Classification Results: In order to test the efficiency of segmentation results obtained by our new method, two main scenarios were designed. The first scenario was planned to understand the effect of using different number of images for the training and testing on the accuracy of the classification when using statistical and texture features. Different combinations between the number of training and testing images are illustrated in Table III.
TABLE III: Various scenarios for evaluating the proposed approach at different training and testing thermograms

\begin{tabular}{|l|l|l|l|l|}
\hline Scenarios & \multicolumn{2}{|l|}{ Training Data } & \multicolumn{2}{l|}{ Testing Data } \\
\hline & Normal & Abnormal & Normal & Abnormal \\
\hline 1st Scenario & 19 & 24 & 10 & 10 \\
\hline 2nd Scenario & 14 & 19 & 15 & 15 \\
\hline 3rd Scenario & 8 & 15 & 21 & 19 \\
\hline 4th Scenario & 4 & 9 & 25 & 25 \\
\hline
\end{tabular}

All the scenarios are evaluated by the SVM functions, i.e. Linear, polynomial, RBF, and quadratic, and the summary of their results are given Table (IV), and Table (V).

The second scenario was designed to investigate whether the integration between the features of texture and statistical is better for detecting the abnormalities in thermograms. Under the same sub-scenarios illustrated in Table (III), the results, evaluated by the accuracy, are shown in Figure (7. The accuracy is calumniated according to Equation (2),

$$
\text { Accuracy }=\frac{T_{P}+T_{N}}{T_{P}+T_{N}+F_{P}+F_{N}}
$$

where $T_{P}$ represents true positive, $T_{N}$ represents true negative, $F_{P}$ represents false positive and $F_{N}$ represents false negative.

\section{DISCUSSION}

As shown in Figure 6, it can be seen that the new segmentation method can successfully extract the ROI (the breasts area) of thermograms. We have some cases where a small part at the bottom of the breast is not included in the extract ROI. However, this part would not affect on the diagnosis of breast abnormality. As reported in [26] that the pectoral region is the most important part for brest cancer diagnosis and it is reported that near $50 \%$ of the breast cancer is located in this region which is successfully extracted by our new segmentation method.

The results of this segmentation method was further evaluated by classifying the segmented ROI to normal and abnormal and the results of different scenario are shown in Tables (IV and V) and Figure (7). From these results, it can be seen that the combination between the statistical and texture features of the ROI gave $100 \%$ which is much better than using each of them individually.

$$
\begin{gathered}
\text { Recall }=\frac{T_{P}}{T_{P}+F_{N}} \\
\text { Precision }=\frac{T_{P}}{T_{P}+F_{P}}
\end{gathered}
$$

The results of the feature combination were further evaluated in terms of the recall and precision described in equation (3) and (4) and the results of these recall and precision are summarized in Figure (8) and Figure (9), respectively. From these two tables and Table (7), it can be noticed that (a) the SVM-RBF gave the best results (100\%) (b) the more training images were used, the high accuracy was obtained as in the first scenario where the accuracy, the recall and the precision reached $100 \%$. These excellent classification results based on 


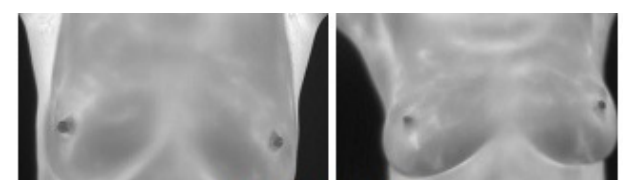

(a)Small breasts

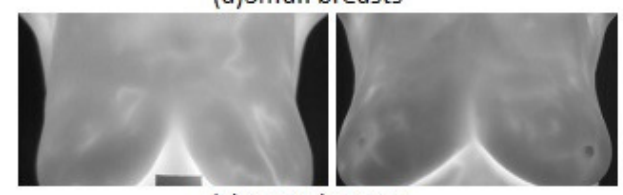

(c) Large breasts

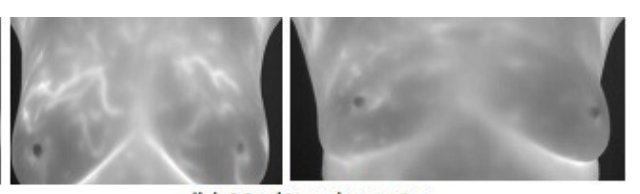

(b) Medium breasts

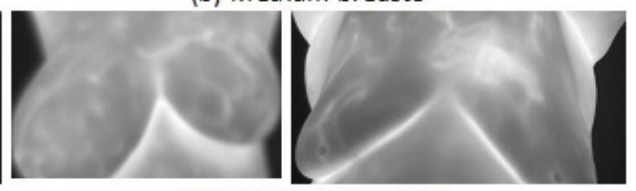

(d) Asymmetric breast

Fig. 6: Different cases Segmentation Results

TABLE IV: Accuracy of the Results of the First Order Statistical Features

\begin{tabular}{|l|l|l|l|l|}
\hline & $\begin{array}{l}\text { First Sce- } \\
\text { nario }\end{array}$ & $\begin{array}{l}\text { Second } \\
\text { Scenario }\end{array}$ & $\begin{array}{l}\text { Third Sce- } \\
\text { nario }\end{array}$ & $\begin{array}{l}\text { Fourth } \\
\text { Scenario }\end{array}$ \\
\hline Quadratic & 85 & 83.33 & 70 & 60 \\
\hline Polynomial & 80 & 66.67 & 70 & 56 \\
\hline RBF & 55 & 60 & 57.14 & 58 \\
\hline Linear & 85 & 76.67 & 72.5 & 58 \\
\hline
\end{tabular}

TABLE V: Accuracy of the Results of Texture Features

\begin{tabular}{|l|l|l|l|l|}
\hline & $\begin{array}{l}\text { First Sce- } \\
\text { nario }\end{array}$ & $\begin{array}{l}\text { Second } \\
\text { Scenario }\end{array}$ & $\begin{array}{l}\text { Third Sce- } \\
\text { nario }\end{array}$ & $\begin{array}{l}\text { Fourth } \\
\text { Scenario }\end{array}$ \\
\hline Quadratic & 80 & 60 & 62.5 & 34 \\
\hline Polynomial & 80 & 56.67 & 47.5 & 50 \\
\hline RBF & 70 & 80 & 65 & 52 \\
\hline Linear & 65 & 56.67 & 62.5 & 50 \\
\hline
\end{tabular}

Accuracy

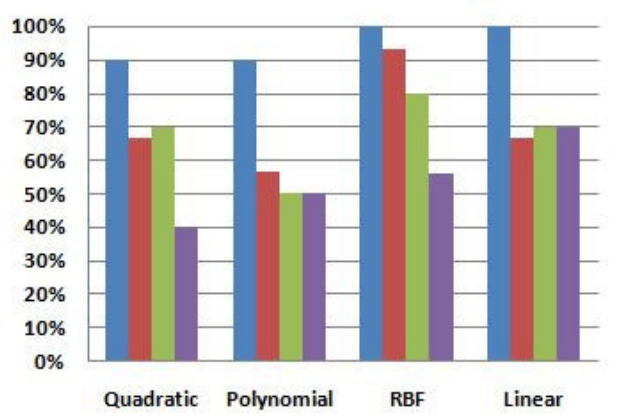

Fig. 7: Accuracy performance measurements our new segmentation method means that this method could be used for the automatic segmentation of the thermal breast cancer images.

\section{CONCLUSION AND FUTURE RESEARCH DIRECTIONS}

In this paper, an automatic segmentation methods for themograms have been proposed. This method is based on the data acquisition protocol parameter (the distance from the patient to the camera) and the image statistics. The proposed segmentation results prove its reliability in extracting the ROI for different cases. This method was evaluated using the segmented ROI in a proposed approach for the detection of the abnormalities of breast thermograms. This approach made

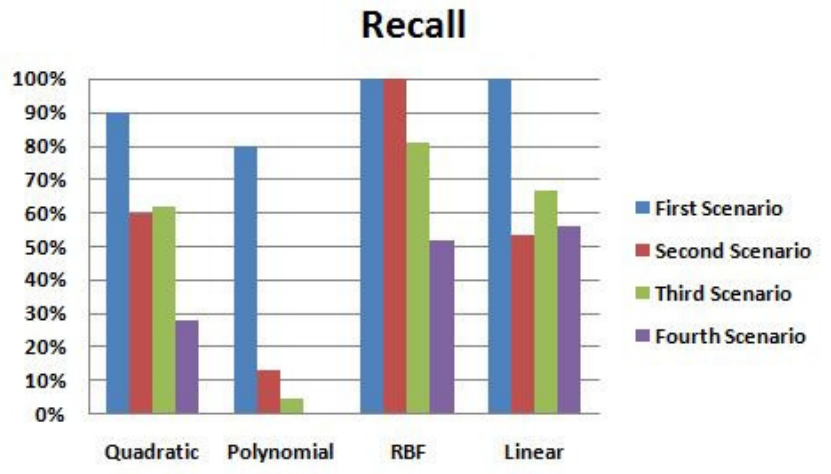

Fig. 8: Recall performance measurements

use of statistical and texture features from the segmented ROI and the SVM with its kernel function was used to detect the normal and abnormal breasts. Based the experimental results, it was found that the SVM-RBF gave the best results (100\%). Also, using the measurements of the recall and the precision, the classification results reached to $100 \%$. These excellent classification results, based on our new segmentation method, concludes that our segmentation method could be used for the automatic segmentation of the thermal breast cancer images. In the future, we plan to increase the dataset used in order to test the reliability of the proposed segmentation method and the classification approach. 


\section{Percision}

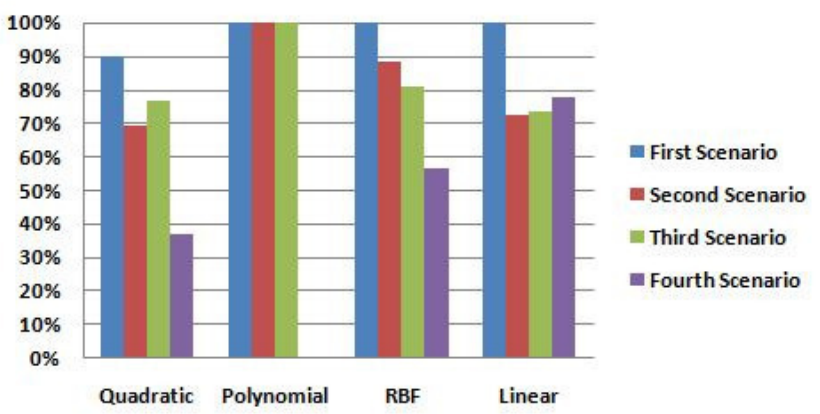

Fig. 9: Percision performance measurements

\section{ACKNOWLEDGMENT}

This paper has been elaborated in the framework of the project "New creative teams in priorities of scientific research", reg. no. CZ.1.07/2.3.00/30.0055, supported by Operational Programme Education for Competitiveness and co-financed by the European Social Fund and the state budget of the Czech Republic and supported by the IT4Innovations Centre of Excellence project (CZ.1.05/1.1.00/02.0070), funded by the European Regional Development Fund and the national budget of the Czech Republic via the Research and Development for Innovations Operational Programme and by Project SP2015/146 "Parallel processing of Big data 2" of the Student Grant System, VSB- Technical University of Ostrava

\section{REFERENCES}

[1] R. Siegel, J. Ma, Z. Zou, and A. Jemal, "Cancer statistics, 2014," CA a cancer journal for clinicians, vol. 64, no. 1, pp. 9-29, 2014.

[2] X. Yao, "A comparison of mammography, ultrasonography, and farinfrared thermography with pathological results in screening and early diagnosis of breast cancer," Asian Biomed, vol. 8, no. 1, 2014.

[3] T. B. Borchartt, A. Conci, R. C. Lima, R. Resmini, and A. Sanchez, "Breast thermography from an image processing viewpoint: A survey," Signal Processing, vol. 93, no. 10, pp. 2785-2803, 2013.

[4] L. Silva, G. Sequeiros, M. L. Santos, C. Fontes, D. C. MuchaluatSaade, and A. Conci, "Thermal signal analysis for breast cancer risk verification," in MEDINFO'15 - 15th World Congress on International Health and Biomedical Informatics, 2015.

[5] N. Arora, D. Martins, D. Ruggerio, E. Tousimis, A. J. Swistel, M. P. Osborne, and R. M. Simmons, "Effectiveness of a noninvasive digital infrared thermal imaging system in the detection of breast cancer," The American Journal of Surgery, vol. 196, no. 4, pp. 523-526, 2008

[6] D. Machado, G. Giraldi, A. Novotny, R. Marques, and A. Conci, "Topological derivative applied to automatic segmentation of frontal breast thermograms," 2013.

[7] S. Suganthi and S. Ramakrishnan, "Semi automatic segmentation of breast thermograms using variational level set method," in The 15th International Conference on Biomedical Engineering. Springer, 2014, pp. 231-234.

[8] S. S. Srinivasan and R. Swaminathan, "Segmentation of breast tissues in infrared images using modified phase based level sets," in Biomedical Informatics and Technology. Springer, 2014, pp. 161-174.
[9] Q. Zhou, Z. Li, and J. K. Aggarwal, "Boundary extraction in thermal images by edge map," in Proceedings of the 2004 ACM symposium on Applied computing. ACM, 2004, pp. 254-258.

[10] L. F. Silva, G. O. S. Olivera, S. Galvao, J. B. Silva, A. A. S. M. D. Santos, D. C. Muchaluat-Saade, and A. Conci, "Análise de séries temporais de sinais térmicos da mama para detecção de anomalias (analysis of time series of breast thermal signs for anomaly detection)," in WIM - XIV Workshop de InformÃatica MĂl'dica. Anais CSBC, 2014, pp. $1818-1827$.

[11] U. R. Acharya, E. Y.-K. Ng, J.-H. Tan, and S. V. Sree, "Thermography based breast cancer detection using texture features and support vector machine," Journal of medical systems, vol. 36, no. 3, pp. 1503-1510, 2012.

[12] T. Jakubowska, B. Wiecek, M. Wysocki, C. Drews-Peszynski, and M. Strzelecki, "Classification of breast thermal images using artificial neural networks," Journal of Medical Informatics \& Technologies, vol. 7 , pp. 41-50, 2004.

[13] S. V. Francis, M. Sasikala, and S. Saranya, "Detection of breast abnormality from thermograms using curvelet transform based feature extraction," Journal of medical systems, vol. 38, no. 4, pp. 1-9, 2014.

[14] M. C. Araújo, R. C. Lima, and R. M. De Souza, "Interval symbolic feature extraction for thermography breast cancer detection," Expert Systems with Applications, vol. 41, no. 15, pp. 6728-6737, 2014.

[15] U. R. Acharya, E. Y.-K. Ng, S. V. Sree, C. K. Chua, and S. Chattopadhyay, "Higher order spectra analysis of breast thermograms for the automated identification of breast cancer," Expert Systems, vol. 31, no. 1 , pp. 37-47, 2014.

[16] S. Suganthi and S. Ramakrishnan, "Analysis of breast thermograms using gabor wavelet anisotropy index," Journal of medical systems, vol. 38, no. 9, pp. 1-7, 2014.

[17] S. Prabha, K. Anandh, C. Sujatha, and S. Ramakrishnan, "Total variation based edge enhancement for level set segmentation and asymmetry analysis in breast thermograms," in Engineering in Medicine and Biology Society (EMBC), 2014 36th Annual International Conference of the IEEE. IEEE, 2014, pp. 6438-6441.

[18] E. Rodrigues, A. Conci, T. Borchartt, A. Paiva, A. C. Silva, and T. MacHenry, "Comparing results of thermographic images based diagnosis for breast diseases," in Systems, Signals and Image Processing (IWSSIP), 2014 International Conference on. IEEE, 2014, pp. 39-42.

[19] A. Tharwat, T. Gaber, and A. E. Hassanien, "Cattle identification based on muzzle images using gabor features and svm classifier," in Advanced Machine Learning Technologies and Applications. Springer, 2014, pp. 236-247.

[20] L. Silva, D. Saade, G. Sequeiros, A. Silva, A. Paiva, R. Bravo, and A. Conci, "A new database for breast research with infrared image," Journal of Medical Imaging and Health Informatics, vol. 4, no. 1, pp. 92-100, 2014.

[21] R. M. Haralick, K. Shanmugam, and I. H. Dinstein, "Textural features for image classification," Systems, Man and Cybernetics, IEEE Transactions on, no. 6, pp. 610-621, 1973.

[22] H. Xu, C. Caramanis, and S. Mannor, "Robustness and regularization of support vector machines," The Journal of Machine Learning Research, vol. 10, pp. 1485-1510, 2009.

[23] A. Tharwat, T. Gaber, A. E. Hassanien, H. A. Hassanien, and M. F. Tolba, "Cattle identification using muzzle print images based on texture features approach," vol. 303, pp. 217-227, 2014.

[24] A. Tharwat, T. Gaber, A. E. Hassanien, M. Shahin, and B. Refaat, "Siftbased arabic sign language recognition system," vol. 334, pp. 359-370, 2015.

[25] N. A. Semary, A. Tharwat, E. Elhariri, and A. E. Hassanien, "Fruitbased tomato grading system using features fusion and support vector machine," in Intelligent Systems' 2014. Springer, 2015, pp. 401-410.

[26] M. Etehadtavakol, E. Ng, V. Chandran, and H. Rabbani, "Separable and non-separable discrete wavelet transform based texture features and image classification of breast thermograms," Infrared Physics \& Technology, vol. 61, pp. 274-286, 2013. 